\title{
L'emploi des expressions épistémiques dans des lettres aux actionnaires en France, aux États Unis et en Allemagne $^{1}$
}

\author{
Svenja Kranich \\ Université de Hambourg, Centre de Recherche Collaboratif sur le Multilingualisme (SFB 538) \\ svenja.kranich@uni-hamburg.de
}

\section{Introduction}

Cette contribution représente une analyse contrastive de l'emploi des expressions de modalité épistémique dans des 'lettres aux actionnaires' (constituant la première partie du rapport annuel) écrites par des sociétés françaises, anglo-américaines et allemandes. Les éléments de modalité épistémique, comme sans doute, cela devrait..., il est possible que... fournissent un moyen linguistique permettant au locuteur d'exprimer que la vérité d'une proposition n'est pas complètement assurée. Une telle stratégie peut être utilisée soit pour des raisons liées au contenu (ne pas avoir accès à suffisamment d'informations), soit pour des raisons interpersonnelles (ne pas vouloir imposer à l'allocuteur un certain point de vue, ne pas vouloir perdre la sympathie de l'allocuteur) (voir Hyland, 1996, 1998). L'emploi d'éléments de ce type représente clairement des avantages, comme lorsqu'on souhaite par exemple souligner que l'on n'exprime qu'une prédiction et non pas une vérité assurée ou lorsqu'on cherche à atténuer l'impact d'une nouvelle négative. Cela peut néanmoins également avoir des inconvénients, étant donné que les auteurs utilisant un grand nombre de ces éléments risquent d'être perçus comme peu crédibles et comme ayant peu confiance en ce qu'ils rapportent, comme l'indiquent les résultats de Crismore et Vande Kopple (1997). Il serait donc intéressant de voir la manière dont les textes provenant de différentes cultures linguistiques varient en ce qui concerne la fréquence et les facteurs influençant l’emploi de ces éléments.

Des différences culturelles entre les pratiques du discours ont été relevées pour la communication économique en général (voir par ex. Clyne, 1987; Bolten et al., 1996; Böttger \& Bührig, 2003, 2004, 2007 ; Baumgarten \& Özçetin, 2008), mais jusqu'à présent peu d'études se sont spécifiquement penchées sur les 'lettres aux actionnaires' (c'est à dire sur le 'mot du P.D.G.' dans le contexte français), du moins en ce qui concerne une perspective contrastive ${ }^{2}$. L'objectif de notre étude est donc de contribuer à une meilleure compréhension des différences entre les normes textuelles et pragmatiques dans ce genre central de la communication économique. Les expressions modales seront au centre de l'étude, car leur appréhension fournit un point de départ permettant de mettre à jour des différences subtiles dans le domaine interpersonnel. Une étude pilote a montré qu'il existe des différences considérables en ce qui concerne la fréquence de ces marqueurs dans les lettres aux actionnaires en Allemagne en comparaison de celles provenant des États Unis (Bicsár \& Kranich, 2009). Notre étude élargit le champ d'investigation aux lettres provenant de France, car des études antérieures ont souligné des différences intéressantes au niveau des stratégies communicatives dans le discours économique français et allemand (Barmeyer \& Demangeat, 2007 ; Bastian \& Hammer, 2007) mais aussi entre le discours économique français et anglais (Jenkins \& Hinds, 1987 ; Böttger \& Bührig, 2003), en ce qui concerne plus particulièrement la relation entre auteur-locuteur et lecteur-allocuteur. De plus, l'étude présente offre une analyse plus détaillée que l'étude pilote, considérant non seulement la fréquence, mais partant aussi à la découverte des facteurs ayant une influence sur l'emploi des expressions épistémiques. L'analyse de leur distribution à travers les catégories grammaticales et sémantiques nous permettra de découvrir des différences quant aux styles communicatifs favorisés dans les trois cultures professionnelles. 


\section{Fonction et structure de la 'lettre aux actionnaires'}

Le type de texte désigné ici 'lettre aux actionnaires' (LAA) ${ }^{3}$ satisfait la fonction d'introduction et de synthèse du rapport annuel. Simultanément, les LAA ont des fonctions de marketing moins visibles: elles ont pour but de donner une image positive de la société aux actionnaires (présents et potentiels) ainsi qu'à d'autres lecteurs potentiels ${ }^{4}$ (voir par ex. les descriptions du genre par Fløttum (1998), Hyland (1998) et Garzone $(2004,2005))$.

On peut dire que la LAA possède un rôle très important, étant la partie la plus fréquemment lue de tout le rapport annuel (Kohut \& Segars, 1992), en particulier en ce qui concerne les lecteurs moins spécialisés (Garzone, $2004: 321$ ). Le bon choix de moyens stylistiques convenables pour valoriser l'image de la société peut donc avoir des répercussions significatives sur le comportement des actionnaires.

La structure de la LAA est comparable dans les trois cultures linguistiques. On y retrouve les trois 'mouvements' (moves dans le sens de Swales (1990)) suivants :

1. Une évaluation de la performance de la société dans l'année passée

2. Un récit des événements et des résultats les plus importants de l'année passée

3. Une déclaration des priorités pour le futur et une prévision de la performance future

(Garzone, 2005 : 194)

Ces 'mouvements' sont présents dans les lettres françaises, anglaises et allemandes. En ce qui concerne leur organisation séquentielle, on peut également observer des traits communs. Premièrement, les trois cultures ont en commun le début typique d'une LAA : en général, la lettre commence avec une évaluation très générale dans laquelle le succès de l'entreprise - ou du moins son succès relatif - est loué (mouvement 1). Deuxièmement, on trouve le récit des événements concrets (mouvement 2) qui, pourtant, est bien souvent entrecoupé d'évaluations. En général, on trouve ici des évaluations plus spécifiques sur les activités diverses conduites par l'entreprise dans l'année. Par exemple, une lettre pourrait, après l'évaluation générale, discuter d'une activité de restructuration de l'entreprise, offrir ensuite des informations sur les divers centres de productions, pour enfin présenter les résultats des efforts faits dans le domaine de la protection de l'environnement. Dans chaque cas, la nouvelle thématique est introduite par une évaluation de l'activité. La conclusion est principalement dédiée aux actions futures, même si parfois, nous retrouvons à nouveau une évaluation générale. On peut dire qu'une structure typique ne montrerait pas la séquence des mouvements 1-2-3, mais plutôt 1-2-1-2-1-2-3, ou bien 1-2-1-2-1-2-3-1-3.

Cette tendance est visible dans les trois cultures professionnelles, mais il existe également des différences interlinguistiques. Ainsi, on peut dire qu'il existe une certaine tendance à présenter plus souvent des commentaires très généraux dans les lettres provenant des États Unis que dans celles provenant de France ou d'Allemagne. Concrètement, la partie dédiée aux mouvements 1 et 3 est plus vaste et l'évaluation aussi bien du passé que du futur reste souvent assez vague dans les lettres anglo-américaines, tandis que le mouvement 2, caractérisé par le récit des événements et des résultats concrets, est représenté dans les trois cultures professionnelles dans la même mesure. Un exemple d'une telle évaluation positive assez vague, qui a presque le caractère d'un slogan, est présenté dans l'exemple suivant :

(1) Our business is more than just our products and services. It's about an experience.

'Notre entreprise ne réside pas seulement dans nos produits et nos services. Il s'agit d'une expérience'.

Le résultat de l'analyse qualitative indique qu'une plus grande importance semble être accordée dans la culture professionnelle américaine à la transmission d'une image positive par des connotations positives et des slogans, tandis que dans les cultures française et allemande, l'accent est plus particulièrement mis sur la description des événements et des résultats concrets. Supposant que la fonction du genre est double (d'un côté, informer sur l'état de l'entreprise, de l'autre, faire de la publicité pour l'entreprise), il est possible de dire que le poids accordé à ces deux fonctions varie selon les cultures professionnelles, les lettres américaines mettant l'accent sur la publicité tandis que les lettres françaises et allemandes 
privilégient l'information. Nous allons voir dans quelle mesure ces différences globales sont reflétées dans la distribution des expressions épistémiques.

\section{L'emploi des expressions épistémiques dans les lettres aux actionnaires}

L'intérêt propre aux expressions épistémiques dans ce genre de texte est surtout lié à leur emploi possible comme moyen d'atténuation ('hedging' dans la littérature anglophone). Dans les analyses récentes, le groupe d'expressions réunis par ce terme est assez vaste. Au début, le terme a été employé pour désigner des éléments tel que a kind of (une sorte de), rather (plutôt) par Lakoff (1972), donc des éléments qui servent à rendre l'appartenance d'un élément à une certaine catégorie moins définitive ('fuzzier'). Depuis les années soixante-dix, le concept de hedging a été élargi pour inclure d'autres éléments réalisant une stratégie communicative qui diminue la responsabilité du locuteur en ce qui concerne la valeur de vérité des propositions (voir Markkanen / Schröder, 1989, 1992). Cela peut être accompli en rendant l'applicabilité d'un terme moins définitive (comme dans le cas de une sorte de, plutôt), mais aussi en signalant une confiance moins que totale dans la vérité de la proposition, et ceci par l'emploi d'un élément modal comme sans doute, probable, devoir (dans son emploi épistémique). La fonction d'atténuation peut aussi être accomplie par des tournures plus longues et plus individuelles qui rendent la proposition vague, comme dans l'exemple suivant :

(2) Aujourd'hui, le Groupe a toutes les cartes en main pour répondre aux enjeux du monde moderne.

Ainsi, il y a, en principe, peu de limites quant aux éléments linguistiques qui peuvent réaliser cette fonction (Markkanen / Schröder, 1997). Le concept d'atténuation étant si vaste, nous avons considéré plus pratique de choisir un groupe particulier et facilement identifiable pour l'analyse parmi les éléments pouvant servir à cette fonction, notamment les expressions epistémiques. Ces expressions peuvent être identifiées en se basant sur la définition suivante : un marqueur épistémique est un moyen linguistique qui indique que le locuteur n'exprime pas une certitude complète concernant la vérité de la proposition $\mathrm{p}$ (Coates, 1995: 59). ${ }^{6}$ Cette définition est basée sur une certaine idéalisation selon laquelle un locuteur n'affirme une proposition non-modalisée que dans le cas où il se sent justifié à croire que la proposition est vraie. ${ }^{7}$ S'il n'est pas sûr de la vérité de la proposition, s'il n'en considère la vérité que comme possible ou probable, il est obligé, par la maxime de qualité de Grice (1975), de marquer en quelque sorte son degré de doute. Ce marquage peut être effectué par des expressions plutôt grammaticales (comme les adverbes modaux tels que probablement ou les verbes modaux tels que may), ou bien par des expressions lexicales (telles que de mon point de vue, nous croyons que). Les critères pour l'extraction de ces marqueurs seront présentés de manière plus détaillée dans la section 4.2.

En nous basant sur les études précédentes portant sur le phénomène de l'atténuation (hedging) dans la LAA (Pindi \& Bloor, 1987 ; Hyland, 1998) et dans d'autres types de textes (par ex. Hyland ,1994, 1996, les articles réunis dans Markkanen \& Schröder (éds.), 1997 ; White \& Sano, 2006), il nous est possible de distinguer deux types d'emploi dans le discours, dépendant de la motivation pour employer un marqueur épistémique:

1. Le locuteur n'a tout simplement pas suffisamment d'informations pour être sûr que la proposition soit vraie, par ex. Il est possible que Martin soit toujours au bureau. Dès lors, ce type sera appelé 'motivation idéationnelle' ${ }^{8}$

2. Le locuteur est tout à fait sûr que la proposition soit vraie, mais il choisit de la présenter de façon moins assurée pour atténuer la force de l'acte de parole ou d'un acte potentiellement menaçant la face de son allocuteur (dans le sens de Brown / Levinson (1987)), par ex. Il est possible qu'il y ait d'autres solutions (que celles que vous avez proposées). Ici, nous nous référerons à ce type comme 'motivation interpersonnelle'.

Il est à supposer que ces deux motivations jouent un rôle dans la LAA. La première motivation peut jouer un rôle surtout dans les contextes du futur - puisque c'est le domaine temporel qui est incertain par sa 
nature même -, tandis que la seconde peut se trouver aussi bien dans les contextes du passé, du présent, et dans ce qu'on a appelé des 'propositions générales' (celles qui font référence à quelque chose plutôt 'hors du temps', par ex. des descriptions de caractéristiques de la société ou du monde en général). Pour classifier les occurrences selon le type de motivation, le critère du cadre de référence peut donc nous fournir un moyen plus objectif que l'intuition seule. Des exemples pour les deux types de motivation sont présentés ci-dessous :

(3) Dans un tel contexte, nous estimons que la marge opérationnelle au premier semestre 2006 devrait se situer dans la ligne du second semestre 2005.

(4) C'est, de mon point de vue, une performance d'autant plus remarquable qu'elle a été réalisée dans un contexte difficile en France, notre premier marché.

Dans l'exemple (3), qui offre une prédiction du développement économique futur de la société, les raisons pour atténuer la proposition se situent dans le domaine idéationnel : il n'est tout simplement pas possible pour le locuteur de connaître le futur. Dans l'exemple (4) par contre, l'auteur se réfère à un succès de l'année passée, et il pourrait également exprimer son évaluation avec une proposition non-modifiée (après tout, il est clair qu'une proposition contenant un attribut évaluatif comme remarquable représente une évaluation subjective). La motivation primaire pour l'emploi de la phrase de mon point de vue dans ce contexte ne semble donc pas être un manque de certitude, mais plutôt une motivation pragmatique, relevant du domaine interpersonnel: le locuteur ne veut pas trop vanter la bonne performance de la société pour ne pas donner une image d'arrogance.

En ce qui concerne la motivation idéationnelle et leur applicabilité dans des propositions orientées vers le futur, il est en effet évident que les sociétés ont une conscience très développée du rôle du langage. Dans la citation suivante, la société donne des informations explicites aux lecteurs sur la manière dont les prédictions faites dans la LAA doivent être comprises :

"These forward-looking statements are not historical facts. Rather, they are based on
the current beliefs, assumptions $[\ldots]$, and projections of the directors [...]. The words
'believe', 'intend', 'expect', 'anticipate', 'project', 'estimate', 'predict' and similar
expressions are intended to identify forward-looking statements."

(Les propositions visant au futur ne sont pas des faits historiques. Elles sont plutôt basées sur des convictions, suppositions, [...] et projections des directeurs [...]. Les mots 'believe' (croire), 'intend' (avoir l'intention), 'expect' (s'atteindre), 'anticipate' (prévoir), 'project' (prévoir), 'estimate' (estimer), 'predict' (prédire) et des expressions similaires sont destinées à identifier des propositions visant au futur.)

(morceau d'une LAA d'une société située à Hong Kong, cité par Bhatia 2005: 50)

Comme nous l'avons déjà mentionné au sujet de l'exemple (4), la motivation interpersonnelle d'employer des marqueurs d'atténuation est liée à la volonté de ne pas apparaître trop arrogant, de laisser le libre choix au lecteur de former sa propre opinion - donc, de ne pas commettre un acte menaçant la face de l'allocuteur. Cet aspect a été souligné dans les analyses du hedging sur le discours académique (voir par ex. Myers, 1989 ; Hyland, 1996). Une autre possibilité d'emploi des éléments épistémiques est celle des 'softener' dans la communication des nouvelles négatives. Étant donné la double fonction de la LAA (d'un côté fournir des informations objectives sur la performance de la société et de l'autre, solidifier la confiance dans la société), on peut penser que ce type d'emploi se retrouve assez fréquemment. On peut ainsi satisfaire aux deux besoins : pour être fidèle à la réalité, il faut inclure des nouvelles négatives le cas échéant mais, en atténuant les propositions respectives, on essaie d'éviter de nuire à l'image de la société. L'exemple suivant illustre ce point :

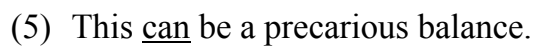

'Cela peut être une équilibre précaire.'

Nous avons choisi pour exemple une lettre anglaise, car ce type d'emploi ne se trouve ni dans notre échantillon de lettres françaises, ni dans celui des lettres allemandes. Même dans les lettres anglaises, il 
est assez rare. Pour expliquer cette rareté, nous pouvons nous appuyer sur l'hypothèse que, dans l'ensemble, les LAA essaient d'éviter des propositions ouvertement négatives, et la modalisation de ce type de propositions n'est pas considérée comme une protection suffisante contre une image négative.

Les différences contrastives intéressantes se trouvent, par contre, dans la fréquence des marqueurs epistémiques vus dans l'ensemble, dans leur distribution à travers les catégories grammaticales, et dans leur emploi au niveau des évaluations positives des sociétés, comme nous le verrons dans la présentation des résultats quantitatifs de l'analyse (section 5). Avant cela, nous allons brièvement présenter le corpus et la méthodologie utilisés pour l'étude présente.

\section{Corpus et méthodologie}

\subsection{Le corpus des LAA}

Le corpus est uniquement constitué de textes du genre LAA, c'est-à-dire du genre de documents se trouvant au début du Rapport Annuel. Il est multilingue et contient, dans sa forme complète, des lettres originales allemandes, américaines, britanniques, françaises et espagnoles. De plus, il contient également des traductions de l'anglais vers l'allemand et vers l'espagnol et de l'allemand vers l'anglais, ce qui permet d'appréhender les effets de traduction (par ex. des interférences entre la langue source et le texte cible). Pour notre étude, seuls des textes originaux (non-traduits) en français, allemand et anglais américain ont été choisis, notre objectif étant d'établir des différences entre les trois cultures linguistiques. Afin de couvrir le champ entier des expressions épistémiques, une lecture proche de tous les textes entiers était nécessaire, car, par la force des choses, une recherche automatisée se serait limitée aux expressions pré-choisies. C'est pour cela que la taille du corpus a été réduite à environ 40.000 mots par langue. Les chiffres exacts sont donnés dans le tableau suivant :

Tableau 1 Le Corpus des Lettres aux Actionnaires

\begin{tabular}{|l|l|}
\hline Langue & Nombre de mots \\
\hline Français & 40.067 \\
Anglais américain & 39.325 \\
Allemand & 39.729 \\
\hline
\end{tabular}

\subsection{Méthodologie}

Afin d'effectuer notre analyse, nous avons isolé tous les cas d'expressions modales épistémiques selon notre définition présentée en section 3. Nous avons donc évalué chaque phrase en nous demandant s'il y a une proposition $\mathrm{p}$ et un élément modal mod qui modifie le degré de certitude avec lequel p est affirmé. Les types d'atténuation qui ne permettent pas une claire distinction entre $\mathrm{p}$ et mod n'ont pas été inclus dans l'analyse. Pour illustrer la différence : dans l'exemple (3), on peut différencier entre p (la marge opérationnelle au premier semestre 2006 se situera dans la ligne du second semestre 2005) et deux éléments servant de mod (nous estimons, devoir : exprimant une haute probabilité que p est vrai). Dans l'exemple (2), par contre, une telle différentiation est difficile : dans le Groupe a toutes les cartes en main pour répondre aux enjeux du monde moderne, c'est la proposition même qui est vague. C'est-à-dire, il est très difficile de savoir sous quelles conditions exactes $\mathrm{p}$ est vraie, mais on ne peut pas dire qu'il y a un élément précis modifiant une proposition qui, sans cet élément, serait présentée comme certaine.

Après avoir ainsi isolé les éléments que nous voulons classifier comme expressions épistémiques, nous avons ensuite classifié ces expressions selon les facteurs suivants : 
1. Classe grammaticale de l'expression

2. Valeur modale de l'expression

3. Référence temporelle de l'expression

4. Référence à la société elle-même (oui ou non)

En ce qui concerne le premier facteur, il peut nous aider à améliorer notre connaissance des différences contrastives entre les trois langues par rapport au champ de modalité épistémique. Le deuxième facteur est d'une importance significative pour une étude qui vise principalement à découvrir des différences culturelles au regard du degré d'atténuation considéré comme adéquat dans ce genre de texte. Nous verrons ici si les éléments d'une faible ou d'une forte valeur de probabilité sont plus fréquents dans les trois langues, et s'il y a des différences. Les éléments exprimant une haute probabilité sont ceux qui favorisent la vérité de $\mathrm{p}$ comparée à des scénarios alternatifs. Si on dit par ex. Nous le ferons probablement, on n'exclut pas les possibilités alternatives, mais elles sont présentées comme moins probables. Donc, on ne peut pas combiner une telle déclaration avec la proposition négative modalisée par le même élément (*Nous le ferons probablement, et probablement nous ne le ferons pas). D'un autre côté, les expressions modales d'une valeur de probabilité basse expriment seulement la possibilité de la vérité de $\mathrm{p}$, sans la favoriser parmi les possibilités alternatives, ce qui permet la combinaison avec la proposition négative modalisée du même élément (par ex. Nous le ferons peut-être, et peut-être nous ne le ferons pas). Ce test de combinaison avec la négation permet la distinction claire et nette de ces deux types de force modale épistémique.

La raison de l'inclusion du facteur de référence temporelle a déjà été expliquée dans la section 3 . Le critère numéro 4, séparant les propositions faisant référence à la société elle-même des autres propositions, a été choisi selon le même raisonnement, à savoir que le locuteur devrait normalement avoir plus d'informations certaines au sujet de la société dont il est directeur (ou dont il est, du moins, un membre suffisamment important pour être impliqué dans la création de ce type de document) que par rapport à d'autres sujets. Donc, nous partons de la supposition que ce sont les modalisations des propositions faisant référence au présent et au passé ainsi qu'à la propre société qui sont les candidats les plus probables pour la catégorie interpersonnelle de l'atténuation, vu que, dans ce contexte, il serait peu probable qu'un réel manque d'informations soit à l'origine de l'emploi d'un élément modal. Par contre, ces contextes se prêtent assez facilement au type d'atténuation visant à maintenir de bonnes relations entre le locuteur et l'allocuteur (par ex. en évitant d'apparaître trop vantard en louant les succès de sa propre société). On pourrait s'imaginer que c'est en particulier ce type d'emploi qui va différer selon les cultures linguistiques, parce que les conceptions de ce qui est approprié dans l'interaction (par ex. quant à la question de savoir en quoi consiste un trop grand orgueil / une trop grande humilité, ou quand il faut être plus ou moins direct) sont divergentes dans les différentes cultures, comme l'ont démontré diverses études de la pragmatique contrastive et de la communication interculturelle (par ex. Byrnes, 1986 ; House, 1996; Kammhuber, 1998; Becker, 2009). On verra dans la section suivante si cela est effectivement le cas.

\section{Résultats quantitatifs}

Les tableaux suivants révèlent des différences entre les trois cultures linguistiques. Commençons avec les différences de la fréquence totale que l'on peut voir dans le tableau 2:

Tableau 2 Fréquence des expressions épistémiques (Nombre absolu des occurrences)

\begin{tabular}{|l|c|}
\hline LAA français & 71 \\
\hline LAA anglo-américains & 103 \\
LAA allemands & 52 \\
\hline
\end{tabular}


On voit ici que les textes anglo-américains emploient le plus grand nombre d'expressions épistémiques, et que les textes allemands contiennent le plus petit nombre. Les textes français se situent au milieu.

En observant la distribution entre les catégories grammaticales, on voit aussi des différences considérables entre les trois langues, comme le montre le tableau suivant :

Tableau 3 Catégorie grammaticale des expressions épistémiques

\begin{tabular}{|l|r|l|l|r|}
\hline & \multicolumn{1}{l}{$\begin{array}{l}\text { Verbes } \\
\text { modaux }\end{array}$} & $\begin{array}{l}\text { Adverbes } \\
\text { modaux }\end{array}$ & \multicolumn{1}{l|}{$\begin{array}{l}\text { Verbes } \\
\text { lexicaux }\end{array}$} & \multicolumn{1}{l}{$\begin{array}{l}\text { Constructions } \\
\text { lexicales }\end{array}$} \\
\hline LAA françaises & $23(32.3 \%)$ & $14(19.7 \%)$ & $8(11.2 \%)$ & $26(36.6 \%)$ \\
LAA anglo-américaines & $19(18.4 \%)$ & $11(10.6 \%)$ & $58(56.3 \%)$ & $15(14.6 \%)$ \\
LAA allemandes & $6(11.5 \%)$ & $7(13.5 \%)$ & $8(15.4 \%)$ & $31(59.6 \%)$ \\
\hline
\end{tabular}

La différence la plus remarquable figure dans la catégorie des verbes lexicaux. Les LAA angloaméricaines les emploient dans plus de la moitié des cas, tandis que dans les lettres françaises et allemandes, la distribution des marqueurs à travers les catégories ne démontre pas une telle préférence. Les expressions employées dans les lettres françaises démontrent une distribution relativement égale à travers les catégories. Les auteurs des lettres allemandes, par contre, choisissent très souvent des expressions appartenant à la catégorie de la construction lexicale.

Considérons maintenant la distribution des éléments épistémiques entre marqueurs de haute et de basse probabilité :

Tableau 4 Fréquence de marqueurs épistémiques de haute et de basse probabilité

\begin{tabular}{|l|l|l|}
\hline & Valeur haute & Valeur basse \\
\hline LAA françaises & $56(77.8 \%)$ & $15(21.1 \%)$ \\
LAA anglo-américaines & $87(84.5 \%)$ & $16(15.5 \%)$ \\
LAA allemandes & $42(80.8 \%)$ & $10(19.2 \%)$ \\
\hline
\end{tabular}

On voit ici que les expressions épistémiques qui attribuent une haute probabilité à la vérité de $\mathrm{p}$ sont beaucoup plus nombreuses dans les trois langues. Les marqueurs qui n'expriment que la possibilité de la vérité de $\mathrm{p}$, sans favoriser $\mathrm{p}$ par rapport aux possibilités alternatives, ont une basse fréquence. La similarité entre les trois langues rend probable le fait qu'il s'agisse d'un trait caractéristique du genre LAA.

La distribution des expressions épistémiques par champ de référence (temporelle et visant la société ou non) démontre, par contre, des différences intéressantes. Étudions d'abord la différence concernant la distribution à travers les catégories de référence temporelle, visualisée dans le tableau suivant :

Tableau 5 Référence temporelle des propositions modalisées

\begin{tabular}{|l|r|r|r|l|}
\hline & \multicolumn{1}{|l|}{ Passé } & Présent & \multicolumn{1}{l|}{$\begin{array}{l}\text { Vérité } \\
\text { générale }\end{array}$} & Futur \\
\hline LAA françaises & $2(2.8 \%)$ & $8(11.3 \%)$ & $12(17.0 \%)$ & $49(69.0 \%)$ \\
LAA anglo-américaines & $4(3.9 \%)$ & $19(18.4 \%)$ & $10(9.7 \%)$ & $70(68.0 \%)$ \\
LAA allemandes & $2(3.9 \%)$ & $5(9.6 \%)$ & $4(7.7 \%)$ & $41(78.8 \%)$ \\
\hline
\end{tabular}


Ce tableau montre d'abord que, dans les trois langues, les LAA modalisent le plus souvent les propositions faisant référence au futur. Environ $70 \%$ des expressions épistémiques dans les lettres françaises et dans les lettres américaines appartiennent à cette catégorie. Dans les LAA allemandes, il s'agit même de presque $80 \%$. On peut donc dire que les lettres françaises et anglo-américaines semblent avoir une tendance un peu plus prononcée à modaliser les propositions faisant référence à des domaines temporels moins liés à l'incertitude que le futur. Dans les lettres françaises, ce sont les propositions à propos des vérités générales qui contribuent à hauteur de près de $20 \%$ à l'ensemble des propositions modalisées; dans les lettres anglo-américaines par contre, ce sont les propositions faisant référence au présent qui représentent la deuxième plus grande catégorie. Pour mieux comprendre la signification de ces résultats, il serait pertinent de les combiner à la catégorie suivante, à savoir le domaine de référence de la proposition, et plus précisément si elle fait référence à la propre société ou non. Le tableau suivant montre les résultats pour le domaine du présent:

Tableau 6 Référence à l'entreprise et à d'autres matières dans le domaine du présent

\begin{tabular}{|c|c|c|}
\hline & $\begin{array}{l}\text { Présent, autres } \\
\text { matières }\end{array}$ & $\begin{array}{l}\text { Présent de } \\
\text { l'entreprise }\end{array}$ \\
\hline LAA françaises & 1 & 7 \\
\hline LAA anglo-américaines & 6 & 13 \\
\hline LAA allemandes & 3 & 2 \\
\hline
\end{tabular}

Dans le domaine du présent, on peut s'apercevoir que les lettres françaises et anglo-américaines se ressemblent dans leur emploi des modalisations: les deux cultures linguistiques emploient la modalisation dans ce domaine temporel surtout en parlant de la propre société. Par contre, les lettres allemandes, qui réservent largement l'emploi des modalisations au domaine du futur, montrent une distribution plus égale dans le domaine du présent entre les propositions à propos de la société et celles qui font référence à d'autres matières. Cela pourrait suggérer que c'est la motivation interpersonnelle qui joue ici un rôle, et que la fonction interpersonnelle est réalisée différemment en allemand que dans les textes français et anglo-américains. Dans la section suivante, nous allons essayer de proposer une explication pour les différences visibles dans les résultats quantitatifs présentés ici.

\section{Discussion des résultats}

Nous avons pu constater dans la section précédente que le degré de similarité entre les trois langues dépend des facteurs considérés. En ce qui concerne la distribution des expressions épistémiques entre les expressions référant à une haute et à une basse probabilité que $\mathrm{p}$ soit vraie, les trois langues montrent une préférence pour les marqueurs de haute probabilité dans le genre étudié. Cela s'explique par une des fonctions centrales de la LAA, notamment celle de transmettre un sentiment de sécurité et de confiance dans la société. Des propositions dont la vérité est présentée comme probable peuvent encore aider à remplir cette fonction, mais l'inclusion d'un grand nombre de propositions dont la vérité est présentée seulement comme possible pourrait aller à contrecourant de ce but.

Tous les autres facteurs montrent des différences quantitatives intéressantes. Abordons d'abord la question des préférences divergentes en ce qui concerne la distribution des marqueurs épistémiques à travers les catégories grammaticales. Nous avons pu constater que le français montre une distribution assez égale, tandis que l'anglais favorise l'emploi de verbes lexicaux et l'allemand, de constructions lexicales. Cependant, il y a une différence entre l'apparente préférence pour une catégorie seule dans l'anglais américain de l'un côté et dans l'allemand de l'autre. En allemand, les constructions lexicales fournissent le plus grand nombre d'exemples à cause d'un haut degré de variation. Ainsi, il y a un nombre considérable d'expressions qui ne se trouvent qu'une seule fois dans notre corpus. Par contre, l'apparente 
préférence pour les verbes lexicaux dans le corpus anglo-américain est le résultat du choix répété d'une seule expression, notamment we believe.

Ce résultat confirme les conclusions d'études précédentes. Böttger et Bührig $(2003,2007)$ présentent des analyses des 'mission statements', un genre de communication économique qui sert à "propager une « image » de l'entreprise parmi les collaborateurs, les actionnaires et également les concurrents » (Böttger \& Bührig, 2007 : 269), donc une fonction qui est partiellement similaire à celles de la LAA. Elles ont trouvé qu'en anglais américain, ce genre a une structure répétitive, commençant chaque paragraphe avec la formule we believe. Dans des traductions d'un tel texte vers le français et vers l'allemand, elles trouvent que la traduction française maintient normalement le parallélisme des débuts des paragraphes, tandis que la traduction allemande diverge du modèle anglais en employant des expressions plus variées pour rendre la même idée. En nous basant sur nos résultats contrastifs, nous pouvons maintenant dire que,dans le cas choisi par Böttger et Bührig (2007), la traduction allemande représente apparemment une adaptation plus effective aux normes du genre dans la culture cible que la traduction française, rendant le texte traduit plus similaire aux textes comparables produits de façon monolingue, car les textes originaux ne semblent pas avoir tendance à répéter le même élément encore et encore, ni en allemand, ni en français. En fait, les textes français et allemands semblent en général plutôt éviter la répétition, suivant l'idéal stylistique de la 'variation élégante', comme l'ont constaté des études antérieures. Ainsi, Vinay et al. (1995) remarquent que le français est plus opposé à la répétition que l'anglais. House (1996, 1997) observe que le discours allemand est plutôt caractérisé par des expressions ad hoc, alors que le discours anglais se sert plus souvent d'expressions toutes faites. Les résultats de Steiner (2008) confirment cette tendance, montrant que le 'type-token ratio' (le rapport entre les signes-types et les signes-occurrences) est différent entre les textes allemands et les textes anglais, les textes allemands étant composés d'un nombre de signes-types plus élevés, indiquant un moindre degré de répétition.

Si on regarde maintenant la distribution différente des expressions épistémiques à travers les champs de référence, on peut lier les résultats à des conclusions présentées dans des études précédentes sur la pragmatique contrastive. Ainsi, la différence entre l'anglais et l'allemand peut être liée aux contrastes généraux en ce qui concerne les préférences communicatives démontrées dans les travaux de House (1996, 1997). Dans ses analyses de divers types de discours, House a pu montrer que l'allemand tend généralement à mettre l'accent sur le domaine idéationnel (dans la terminologie Hallidayenne), donnant un caractère plutôt transactionnel aux textes, tandis que l'anglais favorise un style plus interactionnel, mettant l'accent sur le domaine interpersonnel. En ce qui concerne la modalisation, on peut donc dire que les textes anglais se montrent plus aptes à donner à l'allocuteur l'occasion de former sa propre opinion sans lui imposer de points de vue, ce qui conduit les auteurs à employer fréquemment des expressions modales servant à l'atténuation. Des cas représentant normalement un emploi interpersonnel sont ceux où l'auteur fait référence au domaine temporel du présent et à la propre société, car là, il y a peu de raison d'être incertain de la vérité des propositions. Dans ce contexte, les expressions modales servent souvent à faire apparaître de manière plus modeste une louange des propres activités, comme dans l'exemple suivant :

(6) Perhaps that's why we have one of the lowest staff turnovers in our industry and are rated as one of the world's most attractive employers.

'C'est peut-être pour cela que nous avons une des plus basses fréquences de changement de personnel dans notre industrie et que nous sommes classés parmi les employeurs les plus attrayants du monde'.

Les textes allemands, par contre, cherchent à trouver la façon la plus effective de transmettre les faits importants. Ils n'emploient donc généralement la modalisation que dans les cas où il s'agit de propositions dont la vérité ne peut effectivement pas être assurée (comme c'est le cas pour les prédictions des événements futurs), et moins pour des raisons interpersonnelles. Un exemple de modalisation typique d'une lettre allemande peut être vu dans l'exemple suivant :

(7) Es ist zu erwarten, dass sich auch der Europäische Rat dem Votum des Parlaments anschließen wird. 
'Il est à prévoir que le Conseil Européen se mettra d'accord avec la vote du parlement'.

Les textes français paraissent suivre des conventions se situant au milieu de la concentration angloaméricaine sur le domaine interpersonnel et de la concentration allemande sur le domaine idéationnel. L'emploi des expressions modales dans les contextes du présent et dans les propositions référant à la propre société peut être observé, mais il est moins fréquent que dans les textes anglophones. En ce qui concerne les différences entre l'anglais et l'allemand, le résultat de l'étude présente est donc similaire à celui d'une étude précédente sur la vulgarisation scientifique, comparant des textes originaux en anglais et en allemand avec des traductions de l'anglais en allemand (Kranich, 2009a, 2009b). Cette étude a montré que les textes anglais emploient plus de marqueurs épistémiques que les textes allemands, et que les textes traduits de l'anglais en allemand se situent au milieu, contenant des effets d'interférence de la langue source. Dans les vulgarisations scientifiques aussi, le fait d'employer plus de marqueurs épistémiques a pour effet de créer un texte plus interactionnel, en laissant au lecteur le libre choix de former sa propre opinion sur des faits (souvent sujet à controverse) présentés dans les textes. Ainsi, les résultats convergent et nous amènent à la conclusion que le différent emploi des marqueurs épistémiques reflètent une différente conceptualisation de la relation entre auteur et lecteur dans les langues étudiées, dont l'effet peut être visible dans diverses types de textes.

Il semble plausible de voir dans cela une cause majeure de la différence entre les fréquences absolues des expressions épistémiques dans les trois langues. Dans les trois cultures, on se sert fréquemment d'expressions épistémiques pour marquer un vrai manque d'informations certaines, mais ce sont surtout les textes anglo-américains, avec leur forte focalisation sur le domaine interpersonnel, qui emploient des marqueurs épistémiques assez couramment pour atténuer une proposition qui risquerait autrement d'être interprétée comme arrogante ou vantarde. Cette motivation a moins d'influence sur les textes français, et encore moins sur les textes allemands, qui privilégient la transmission effective des informations.

\section{Conclusion}

Dans la présente contribution, nous avons présenté de manière détaillée quelques facteurs responsables de la variation interlinguistique dans un type de texte central de la communication économique. L'analyse des lettres aux actionnaires (constituant la première partie du rapport annuel) provenant des sociétés françaises, allemandes et anglo-américaines a démontré que l'emploi des éléments épistémiques est différent selon la culture professionnelle (France, Allemagne et États-Unis) et ce, sur plusieurs aspects. Premièrement, on a pu constater que la fréquence avec laquelle ces éléments se trouvent dans les textes est plus élevée dans les textes anglo-américains que dans les textes français, tandis que ceux-ci les emploient plus souvent que les textes allemands. Nous avons proposé comme explication pour cette divergence les différences de normes textuelles dans le domaine interpersonnel. Alors que les textes allemands tendent généralement à privilégier la transmission effective des informations ( en se focalisant sur le domaine idéationnel), les textes anglo-américains attachent plus d'importance à la création d'une bonne relation interpersonnelle entre locuteur et allocuteur. Ce résultat est aussi en accord avec le résultat de l'analyse textuelle qualitative, dans laquelle nous avons pu également trouver des indices sur le fait que les LAA anglo-américaines mettent l'accent sur l'établissement d'une image positive, tandis que les LAA allemandes et françaises mettent quant à elles l'accent sur l'information concernant des événements concrets. En ce qui concerne les éléments épistémiques, nous avons vu qu'ils sont employés dans les LAA américaines non seulement dans les contextes où la connaissance des faits n'est pas suffisante pour permettre une proposition plus assurée (comme c'est le cas lorsqu'on prévoit des événements futurs), mais aussi dans les contextes où il n'est normalement pas question d'un vrai manque d'informations. L'évidence pour ces tendances différentes peut être vue dans la distribution différente des expressions épistémiques entre les différents domaines temporels et entre les propositions se référant à la propre société et celles faisant référence à d'autres matières : tandis que les propositions modalisées en allemand font généralement référence aux événements futurs, les propositions modalisées dans les textes anglais sont aussi employées dans le contexte du présent et en faisant référence à la société elle-même. De telles modalisations servent communément à complimenter sa propre société de façon atténuée pour éviter de paraitre trop vantard. Quant à cet aspect, les textes français se situent au milieu entre les conventions 
anglo-américaines et les conventions allemandes : ils emploient ce type de modalisation interpersonnelle de temps en temps, mais moins fréquemment que les textes anglo-américains.

En ce qui concerne la distribution des expressions épistémiques à travers les catégories linguistiques, nous sommes arrivés au résultat que l'emploi fréquent d'éléments appartenant à la catégorie de verbe lexical en anglais-américain est l'effet de la répétition fréquente de l'expression we believe dans ce genre de texte. Ni les lettres françaises, ni les lettres allemandes ne montrent une telle préférence pour une seule expression, un contraste que nous avons lié à des résultats d'analyses stylistiques contrastives précédentes, qui montrent que le français ainsi que l'allemand considèrent plus élégant un style varié, évitant des répétitions, alors que l'anglais ne semble pas suivre une telle maxime.

Ces résultats sont révélateurs des différences stylistiques et pragmatiques entre les trois cultures, du moins en ce qui concerne le type de texte analysé, les lettres aux actionnaires, et indiquent peut-être aussi des différences assez générales dans les conventions discursives. De tels résultats peuvent améliorer notre connaissance des contrastes entre les différentes conventions textuelles, et seraient à intégrer dans l'enseignement des langues étrangères, où l'enseignement de la communication économique et des différences interculturelles joue un rôle de plus en plus important (voir p.ex. Spillner, 2007 : 138).

\section{Références bibliographiques}

Barmeyer, C. et Demangeat, I. (2007). Notions partagées ou malentendus interculturels ? Regards sur quelques «mots-clés » dans les coopérations managériales franco-allemandes. Behr et al. (éds), 371-391.

Bastian, S. et Hammer, F. (2007). Commentaire boursier et subjectivité. Étude contrastive français-allemand de messages sur Internet. Behr et al. (éds), 349-365.

Baumgarten, N. et Özçetin, D. (2008). Linguistic variation through language contact in translation. Siemund, P. et N. Kintana (éds.), Language Contact and Contact Languages. Amsterdam: Benjamins, 293-316.

Becker, A. (2009). Modality and engagement in British and German political interviews. Languages in Contrast 9, 522.

Behr, I., Hentschel, D. et Kauffman, M. (éds.), Langue, économie, entreprise. Le travail des mots. Paris : Presses Sorbonne Nouvelle.

Bhatia, V.K. (2005). Interdiscursivity in business letters. Gillaerts et Gotti (éds.), 31-54.

Bicsár, A. et Kranich, S. (2009). These forecasts may be substantially different from actual results. The use of epistemic modal markers in letters to shareholders in English compared to their German translations and original German letters to shareholders. Workshop Multilingual Discourse Production, Hambourg, 06.-07.11.2009.

Bolten, J., Dathe, M., Kirchmeyer, S., Roennau, M., Witchalls, P. et Ziebell-Drabo, S. (1996). Interkulturalität, Interlingualität und Standardisierung bei der Öffentlichkeitsarbeit von Unternehmen. Baumann, K.-D. et H. Kalverkämper (éds.), Fachliche Textsorten. Tübingen : Narr, 389-425.

Böttger, C. et Bührig, K. (2003). Translating obligation in business communication. Perez Gonzales, L. (éd.), Speaking in Tongues: Language across Contexts and Users. Valencia : Université de Valencia, 161-182.

Böttger, C. et Bührig, K. (2007). La communication économique et les traductions. Behr et al. (éds), 269-283.

Brown, P. and Levinson S. (1987). Politeness: Some Universals in Language Usage. (réimprimé avec une nouvelle introduction par E. Goody). Cambridge: Cambridge University Press.

Byrnes, H. (1986). Interactional style in German and American conversations. Text 2, 189-207.

Clyne, M. (1987). Cultural differences in the organization of academic texts. English and German. Journal of Pragmatics 11, 211-247.

Coates, J. (1995). The expression of root and epistemic possibility in English. Bybee, J. et S. Fleischmann (éds.) Modality in Grammar and Discourse. Amsterdam: Benjamins, 55-66.

Crismore, A. et Vande Kopple, W. J. (1997). Hedges and readers: Effects on attitudes and learning. Markkanen et Schröder (1997) (éds.), 83-113. 
Fløttum, K. (1998). Le Mot du P.D.G. - descriptif ou polémique ? Gambier, Y. (éd.), Discours professionnels en Français. Frankfurt : Peter Lang, 105-122.

Fløttum, K., Hemmingsen, I. et Pereira, U.P. (1994). Readability in English, French and German 'Chairman's Statements’. Brekke, M., Ø. Andersen, T. Dahl et J. Myking (éds.), Applications and Implications of Current LSP Research. Vol. II. Bergen : Fagbokforlaget, 729-737.

Garzone, G. (2004). Annual Company Reports and CEO's Letters: Discoursal features and cultural markedness. Candlin, C.N. et M. Gotti (éds.), Intercultural Aspects of Specialized Communication. Bern : Lang, 311-341.

Garzone, G. (2005). Letters to Shareholders and Chairman's Statements: Textual variability and generic integrity. Gillaerts et Gotti (éds.), 179-204.

Gillaerts, P. et Gotti, M. (éds.) (2005). Genre Variation in Business Letters. Bern: Lang.

Gosselin, L. (2010). Les modalités en français. La validation des représentations. Amsterdam : Rodopi.

Grice, H.P. (1975). Logic and conversation. Cole, P. et J.L. Morgan (éds.), Syntax and semantics, tome 3 Speech Acts, New York, Academic Press, p. 41-58.

Halliday, M.A.K. et Matthiessen, C. (2004). An Introduction to Functional Grammar. 3 éd. London : Arnold.

House, J. (1996). Contrastive discourse analysis and misunderstanding: The Case of German and English. Hellinger, M. et U. Ammon (éds), Contrastive Sociolinguistics. Berlin : Mouton, 345-361.

House, J. (1997). Translation Quality Assessment. A Model Revisited. Tübingen: Narr.

Hyland, K. (1994). Hedging in academic writing and EAP textbooks. English for Specific Purposes 13, 239-256.

Hyland, K. (1996). Writing without conviction? Hedging in science research articles. Applied Linguistics 17, 433454.

Hyland, K. (1998). Boosting, hedging, and the negotiation of academic knowledge. TEXT 18, 349-382.

Jenkins, S. et Hinds, J. (1987). Business letter writing: English, French and Japanese. TESOL Quaterly 21, 327-349.

Kammhuber, S. (1998). Kulturstandards in der interkulturellen Kommunikation: Grobe Klötze oder nützliche Denkgriffe? Jonach, I. (éd.). Interkulturelle Kommunikation. München : Ernst Reinhardt Verlag, 27-36.

Kauffmann, M. (2007). La présentation de l'entreprise dans les rapports annuels français et les Geschäftsberichte/Jahresberichte allemands. Behr et al. (éds), 249-266.

Kohut, G.F. et Segars, A.H. (1992). The President's Letter to Stockholders: An Examination of Corporate Communication Strategies. The Journal of Business Communication 29, 7-21.

Kranich, S. (2009a). Epistemic modality in English popular scientific articles and their German translations. transkom. Zeitschrift für Translationswissenschaft und Fachkommunikation 2 [1]: 26-41.

Kranich, S. (2009b). Epistemic modality between marker of true uncertainty and politeness strategy. Evidence from English and German popular scientific writing. Papier présenté à l'occasion de la $11^{\mathrm{e}}$ IPra (International Pragmatics Association), Melbourne, 12-17.07.2009.

Küppers, A. (2008). Sprecherdeiktika in deutschen und französischen Aktionärsbriefen. [Arbeiten zur Mehrsprachigkeit 87 (Série B)]. Hamburg : Universität Hamburg.

Lakoff, G. (1972). Hedges: A study of meaning criteria and the logic of fuzzy concepts. Peranteau, P., J. Levi et G. Phares (éds.), Papers from the Eighth Regional Meeting of Chicago Linguistic Society. Chicago : Chicago University Press, 183-228.

Markkanen, R. et Schröder, H. (1989). Hedging as a translation problem in scientific texts. C. Laurén et M. Nordman (éds.), Special Language: From Humans Thinking to Thinking Machines. Clevedon: Multilingual Matters, 171175.

Markkanen, R. et Schröder, H. (1992). Hedging and its linguistic realization in English, German and Finnish philosophical texts: A case study. M. Nordman (éd.), Fachsprachliche Miniaturen. Frankfurt/Main : Peter Lang, $121-130$.

Markkanen, R. et Schröder, H. (éds.). (1997). Hedging and Discourse. Approaches to the Analysis of a Pragmatic Phenomenon in Academic Texts. Berlin: Walter de Gruyter. 
Myers, G. (1989). The pragmatics of politeness in scientific articles. Applied Linguistics 10, 1-35.

Nickerson, C. et De Groot, E. (2005). Dear Shareholder, Dear Stockholder, Dear Stakeholder : The business letter genre in the Annual General Report. Gillaerts et Gotti (éds.), 324-345.

Pindi, M. et Bloor, T. (1987). Playing safe with predictions: Hedging, attribution and conditions in economic forecasting. Bloor, T. et J. Norrish (éds.), Written Language, CILT, 55-69.

Roulet, E., Filliettaz, L., Grobet, A. et Burger, M. (2001). Un modèle et un instrument d'analyse de l'organisation du discours. Bern: Peter Lang.

Spillner, B. (2007). Discours économique: Variétés intralinguistiques et différences contrastives. Behr et al. (éds.), 117-145.

Steiner, E. (2008). Empirical studies of translations as a mode of language contact. 'Explicitness' of lexicogrammatical encoding as a relevant dimension. Siemund, P. et N. Kintana (éds.), Language Contact and Contact Languages. Amsterdam : Benjamins, 317-345.

Suomela-Salmi, E. et Gambier, Y. (1994). Le Mot du PDG : un leurre franco-finlandais. Moirand, S., Ali Bouacha A.A., Beacco J.C. et Collinot A. (éds.), Parcours linguistiques de discours spécialisés. Bern : Peter Lang, 219230.

Swales, J.M. (1990). Genre Analysis: English in Academic and Research Settings. Cambridge : Cambridge University Press.

Vinay, J.-P., Darbelnet, J., Sager, J.C. et Hamel, M.-J. (1995). Comparative stylistics of French and English: a methodology for translation. Amsterdam : Benjamins.

White, P.R.R et Sano, M. (2006). Dialogistic positions and anticipated audiences - a framework for stylistic comparisons. Aijmer, K. et A.-M. Simon-Vandenbergen (éds.), Pragmatic Markers in Contrast. Amsterdam: Elsevier, 189-214

${ }^{1}$ Je voudrais remercier Andrea Bicsár pour la collaboration agréable à une étude pilote qui a précédé la présente investigation (Bicsár \& Kranich, 2009), Anne Küppers pour me donner accès au corpus des LAA françaises qu'elle a compilé pour sa thèse de Maîtrise, Martin Elsig pour sa relecture d'une version précédente de ce texte et pour ses corrections qui m'ont été d'une grande aide, et Aurélie Denoyer pour ses corrections très efficaces et professionnelles.

${ }^{2}$ Notez les exceptions suivantes : Suomela-Salmi et Gambier (1994) ont présenté une analyse contrastive du genre, comparant des lettres françaises et finlandaises ; Fløttum, Hemmingsen, Pereira et Unni (1994) comparent les mêmes langues que celles présentes dans notre étude se concentrant sur la question de l'aise de lecture (readability). Nickerson et De Groot présentent quelques résultats sur l'organisation discursive des LAA provenant des Pays Bas et de la Grande Bretagne. Garzone (2004) fait une comparaison entre les lettres aux actionnaires italiennes et les lettres émanant d'autres pays européens et des États Unis. Bien qu'elle arrive ainsi à livrer des résultats intéressants sur les caractéristiques spécifiques du genre en Italie, il aurait été souhaitable dans une perspective contrastive de faire une analyse distincte de chaque groupe national plutôt qu'une comparaison entre les textes italiens d'un côté et les textes provenant de toutes les autres cultures linguistiques dans l'ensemble de l'autre. Finalement, Küppers (2008) a présenté une analyse pertinente de l'emploi des pronoms personnels dans des LAA françaises et allemandes. En ce qui concerne le rapport annuel (contenant la LAA ainsi que des documents divers), on trouve une comparaison de la présentation de l'entreprise dans des rapports annuels français et allemands dans Kauffmann (2007), qui met l'accent sur des lexèmes / des idiomes choisis représentant les valeurs des entreprises.

${ }^{3}$ Ce qu'on appelle ici 'la lettre aux actionnaires' est plus communément désigné par le terme 'le mot du P.D.G.' dans les études portant sur le terrain français. Comme il s'agit ici d'une analyse contrastive (et l'organisation des sociétés diffère entre les pays), on a choisi d'employer ce terme, plus général.

${ }^{4}$ Comme ces documents sont, de nos jours, généralement publiés sur internet, ils sont accessibles à un grand nombre de personnes. Le public visé comme lecteur-allocutaire principal est néanmoins constitué d'actionnaires actuels et de professionnels vendeurs des titres dans les banques.

${ }^{5}$ Les résultats de notre analyse laissent supposer qu'une analyse séquentielle plus détaillée de ce type de texte, adoptant pour ce but, par exemple, le modèle proposé par Roulet et al. (2001), pourrait produire des résultats très éclairants. Dans une telle analyse du discours, on pourrait également prendre en compte l'organisation hiérarchique des séquences. Dans l'analyse présente, l'analyse qualitative du discours se limitera cependant à quelques 
observations générales sur la structure discursive, car notre focale est mise sur les résultats quantitatifs quant à l'emploi des marqueurs épistémiques.

${ }^{6}$ Notre délimitation du champ de modalité épistémique est donc plus restrainte que celle de Gosselin (2010: 325331), car, par définition, elle n'inclut pas de cas de modalité véridicible.

${ }^{7}$ D'un point de vue philosophique, la notion de vérité est assez problématique. Ici, le terme 'vérité' est employé pour dénoter la vérité subjective du locuteur. Ce qui décide de l'emploi d'un élément modal est la croyance du le locuteur en ce que la proposition soit vraie (alors il peut employer le modus assertif, non-modalisé) ou en ce que la vérité de la proposition soit seulement probable ou possible (alors il est forcé d'employer une modalisation).

${ }^{8}$ Le choix terminologique est inspiré par la distinction faite dans la grammaire systémique entre domaine idéationnel, interpersonel et textuel (Halliday \& Matthiessen, 2004). 